\title{
Ion structure factors and electron transport in dense Coulomb plasmas*
}

\author{
D.A. Baiko, A.D. Kaminker, A.Y. Potekhin, and D.G. Yakovlev \\ Ioffe Physical-Technical Institute, Politekhnicheskaya 26, 194021 St.-Petersburg, Russia
}

(Received 21 August 1998)

The dynamical structure factor of a Coulomb crystal of ions is calculated at arbitrary temperature below the melting point taking into account multi-phonon processes in the harmonic approximation. In a strongly coupled Coulomb ion liquid, the static structure factor is split into two parts, a Bragg-diffraction-like one, describing incipient long-range order structures, and an inelastic part corresponding to thermal ion density fluctuations. It is assumed that the diffractionlike scattering does not lead to the electron relaxation in the liquid phase. This assumption, together with the inclusion of multi-phonon processes in the crystalline phase, eliminates large discontinuities of the transport coefficients (jumps of the thermal and electric conductivities, as well as shear viscosity, reported previously) at a melting point.

PACS numbers: 52.25.Fi, 95.30.Qd, 97.20.Rp, 97.60.Jd

We consider a strongly coupled Coulomb plasma (SCCP) of ions immersed in a nearly uniform chargecompensating electron gas. The ions may be disordered (liquid phase) or arranged in a crystalline lattice. The energetically favorable body-centered cubic (bcc) lattice, appears at $\Gamma>\Gamma_{m} \approx 172$ [1], where $\Gamma=(Z e)^{2} /(a T)$ is the ion-coupling parameter, $T$ is the temperature, $a=\left(4 \pi n_{i} / 3\right)^{-1 / 3}$, and $n_{i}$ is the ion number density.

Many astrophysical objects (interiors of white dwarfs, massive stars, and giant planets; envelopes of neutron stars) are made of such a plasma. Its kinetic properties required for various applications are determined mainly by electron-ion (ei) scattering. A general framework for calculation of these transport properties has been described in [2]. Numerous calculations (e.g., [3]), done under additional assumption of strong electron degeneracy, predict large (a factor of 3-4) discontinuities of the electric and thermal conductivities at the melting point. In contrast, the thermodynamic quantities in the liquid and solid phases, determined solely by ions, are very similar near $\Gamma=\Gamma_{m}$ (e.g., 8[1]). This suggests that properties of the ion system serving as a main scatterer for electrons should vary smoothly through the melting transition. In this Letter, we propose a modification of the transport theory which removes large jumps of the transport coefficients.

The differential ei scattering rate in a SCCP averaged over initial and summed over final electron spin states $\sigma$

*Phys. Rev. Lett. 81, No. 24 (14 December 1998) and $\sigma^{\prime}$ is

$$
\begin{aligned}
\Gamma\left(\mathbf{p} \rightarrow \mathbf{p}^{\prime}\right)= & \frac{2 \pi N}{\hbar^{2}} \frac{1}{2} \sum_{\sigma \sigma^{\prime}}\left|U_{\mathbf{q}, \sigma^{\prime} \sigma}\right|^{2} \mathcal{S}(\mathbf{q}, \omega), \\
\mathcal{S}(\mathbf{q}, \omega)= & \frac{1}{2 \pi} \int_{-\infty}^{+\infty} \mathrm{d} t e^{-i \omega t} S(\mathbf{q}, t) \\
= & \frac{1}{2 \pi N} \int_{-\infty}^{+\infty} \mathrm{d} t \int \mathrm{d} \mathbf{x} \mathrm{d} \mathbf{x}^{\prime} e^{i \mathbf{q} \cdot\left(\mathbf{x}-\mathbf{x}^{\prime}\right)-i \omega t} \\
& \times\left\langle\hat{\rho}^{\dagger}(\mathbf{x}, t) \hat{\rho}\left(\mathbf{x}^{\prime}, 0\right)\right\rangle_{T},
\end{aligned}
$$

where $N$ is the total number of ions, $\mathbf{p}$ and $\mathbf{p}^{\prime}$ are the electron momenta before and after scattering, respectively, $\hbar \mathbf{q}=\mathbf{p}^{\prime}-\mathbf{p}, \hbar \omega=\epsilon^{\prime}-\epsilon$ is the difference between final and initial electron energies, and $U_{\mathbf{q}, \sigma^{\prime} \sigma}$ is the matrix element of the operator of elementary $e i$ interaction. $\mathcal{S}(\mathbf{q}, \omega)$ is the dynamical structure factor of the plasma, the most important quantity of the theory. In the liquid regime, $\hat{\rho}(\mathbf{x}, t)$ is the operator of the charge density in units of $Z|e|: \hat{\rho}(\mathbf{x}, t)=\hat{\mathrm{n}}_{\mathrm{I}}(\mathbf{x}, t)-n_{i}$, where $\hat{\mathrm{n}}_{\mathrm{I}}(\mathbf{x}, t)$ is the ion density operator and $n_{i}=n_{e} / Z$ takes account of the compensating electron background with the electron density $n_{e}$. In the solid regime, $\hat{\rho}(\mathbf{x}, t)=\hat{\mathrm{n}}_{\mathrm{I}}(\mathbf{x}, t)-\sum_{i} \delta\left(\mathbf{x}-\mathbf{R}_{i}\right)$ (where $\mathbf{R}_{i}$ is a lattice vector), i.e. the operator of fluctuations of the charge density.

Integrating over $\mathbf{x}$ and $\mathbf{x}^{\prime}$ we obtain the structure factor of the ion density fluctuations in the solid phase in the form

$$
\begin{gathered}
N S_{\mathrm{sol}}(\mathbf{q}, t)=\left\langle\sum_{i, j} e^{i \mathbf{q} \cdot\left(\mathbf{R}_{i}-\mathbf{R}_{j}\right)}\left[e^{i \mathbf{q} \cdot \mathbf{u}_{i}(t)}-1\right]\right. \\
\left.\times\left[e^{-i \mathbf{q} \cdot \mathbf{u}_{j}(0)}-1\right]\right\rangle_{T}
\end{gathered}
$$

where $\mathbf{u}_{i}$ is an ion displacement from $\mathbf{R}_{i}$. Expanding $\mathbf{u}_{i}$ in the phonon normal coordinates and using the Weyl operator identity $e^{A} e^{B}=e^{A+B} e^{[A, B] / 2}$, we can decompose $S_{\text {sol }}(\mathbf{q}, t)$ into the elastic (Bragg) and inelastic parts $S_{\mathrm{sol}}(\mathbf{q}, t)=S_{\mathrm{sol}}^{\prime}(\mathbf{q})+S_{\mathrm{sol}}^{\prime \prime}(\mathbf{q}, t)$. The elastic part is easily calculated (cf. [9]):

$$
S_{\text {sol }}^{\prime}(\mathbf{q})=\left(1-e^{-W}\right)^{2}(2 \pi)^{3} n_{i} \sum_{\mathbf{G}} \delta(\mathbf{q}-\mathbf{G}),
$$

where $\mathbf{G}$ is a reciprocal lattice vector, and $W=W(\mathbf{q})$ is the Debye-Waller factor, $\exp (-W)=\left\langle\exp \left(i \mathbf{q} \cdot \mathbf{u}_{j}\right)\right\rangle_{T}$,

$$
W=\frac{\hbar}{2 M N} \sum_{\nu} \frac{\left(\mathbf{q} \cdot \mathbf{e}_{\nu}\right)^{2}}{\omega_{\nu}}\left(\bar{n}_{\nu}+\frac{1}{2}\right) .
$$


In this case, $M$ is the ion mass, $\nu \equiv(\mathbf{k}, s), s=1,2,3$ enumerates phonon modes, $\mathbf{k}$ is a phonon wavevector, $\mathbf{e}_{\nu}$ the polarization vector, $\omega_{\nu}$ the frequency, and $\bar{n}_{\nu}=$ $\left(e^{z_{\nu}}-1\right)^{-1}$ is the mean number of phonons, $z_{\nu}=\hbar \omega_{\nu} / T$. For the lattice types of interest (e.g., bcc), $W=r_{T}^{2} q^{2} / 6$, where $r_{T}^{2}=\left\langle\mathbf{u}^{2}\right\rangle_{T}$ is the mean-squared ion displacement (cf. 6, 6 ).

The Bragg scattering of electrons results in the energy band structure of the electron Bloch states, but does not contribute to the ei collision integral in the kinetic equation [2]. Indeed, this scattering occurs at the boundaries of the Brillouin zones and translates an electron from one zone to another. The transition requires change of the electron energy by the value of the interband gap; thus another particle must be involved to carry the excess energy.

Therefore only the inelastic part of the structure factor contributes to the collision integral. The inelastic part can be found by the same technique [9]:

$$
\begin{aligned}
& N S_{\text {sol }}^{\prime \prime}(\mathbf{q}, t)=e^{-2 W} \sum_{i j} e^{i \mathbf{q} \cdot\left(\mathbf{R}_{i}-\mathbf{R}_{j}\right)} \sum_{n=1}^{\infty} \frac{1}{n !} \\
& \times\left\{\frac{\hbar}{2 M N} \sum_{\nu} \frac{\left(\mathbf{q} \cdot \mathbf{e}_{\nu}\right)^{2}}{\omega_{\nu}}\left[\alpha_{i j \nu}\left(\bar{n}_{\nu}+1\right)+\alpha_{i j \nu}^{*} \bar{n}_{\nu}\right]\right\}^{n},
\end{aligned}
$$

where $\alpha_{i j \nu} \equiv \exp \left[i \mathbf{k} \cdot\left(\mathbf{R}_{i}-\mathbf{R}_{j}\right)-i \omega_{\nu} t\right]$. The summation over $i, j$ yields the delta function which removes one summation over $\mathbf{k}$ (included in the sums over $\nu$ ). Thus we have $n$ sums over $s$ and $n-1$ sums over $\mathbf{k}$ in each $n$th term of Eq. (6).

Retaining the first term $n=1$, we recover the onephonon approximation employed in previous works (e.g., [2] 6]). Our point is that this approximation fails near the melting point. In fact, the contribution of the $n$-phonon processes ( $n$th term) at $T$ above the Debye temperature can be estimated as $\left(q r_{T}\right)^{2 n} / n ! \sim\left(k_{F} r_{T}\right)^{2 n} / n$ !, where $k_{F}=\left(3 \pi^{2} n_{e}\right)^{1 / 3}$ is the electron Fermi wave number, $r_{T}^{2} \approx$ $u_{-2} a^{2} / \Gamma, u_{-2} \equiv\left\langle\omega_{p}^{2} / \omega_{\nu}^{2}\right\rangle_{\mathrm{ph}} \approx 13$ is a frequency moment for a bcc lattice [8], $\omega_{p}$ is the ion plasma frequency, and $\langle\ldots\rangle_{\mathrm{ph}}$ denotes averaging over phonon spectrum (e.g., [6]) in the harmonic-lattice approximation. For instance, for Fe plasma at $\Gamma \approx \Gamma_{m}$ we obtain a typical value of $n \sim$ $\left(k_{F} r_{T}\right)^{2} \approx 3$, which is not small.

An important difference of astrophysical Coulomb crystals from the terrestrial metals is that the umklapp processes dominate the scattering, because the equivalent radius of the Brillouin zone $q_{B}=\left(6 \pi^{2} n_{i}\right)^{1 / 3}$ is smaller than typical momentum transfers $q \sim k_{F}$. For $q>$ $q_{B}$ one can approximately replace $\sum_{s}\left(\mathbf{q} \cdot \mathbf{e}_{\nu}\right)^{2} f\left(\omega_{\nu}\right) \rightarrow$ $q^{2}\left\langle f\left(\omega_{\nu}\right)\right\rangle_{\mathrm{ph}}[10]$. Then the remaining summations in Eq. (6) are done explicitly:

$$
\begin{aligned}
& e^{2 W} S_{\mathrm{sol}}^{\prime \prime}(\mathbf{q}, t)= \\
& \exp \left[\frac{\hbar q^{2}}{2 M}\left\langle\frac{\cos \left(\omega_{\nu} t\right)}{\omega_{\nu} \tanh \left(z_{\nu} / 2\right)}-i \frac{\sin \left(\omega_{\nu} t\right)}{\omega_{\nu}}\right\rangle_{\mathrm{ph}}\right]-1
\end{aligned}
$$

The static structure factor is defined as $S(\mathbf{q})=$ $\int_{-\infty}^{+\infty} \mathcal{S}(\mathbf{q}, \omega) \mathrm{d} \omega=S(\mathrm{q}, 0)$. Thus the inelastic contribution to $S(\mathbf{q})$ in the solid is given by setting $t=0$ in Eq. (7), which yields $S_{\text {sol }}^{\prime \prime}(\mathbf{q})=1-e^{-2 W}$.

Let us turn to the liquid phase. Numerical simulations by different authors show the appearance of incipient long-range order at $\Gamma \gg 1$. For example, Schmidt et al. [11 observed a shear mode at $\Gamma>100$ in their molecular-dynamics experiment along with the familiar longitudinal ion plasmon. We have verified that the spectrum of these modes can be described by the phonon spectrum averaged over orientations of a crystal. Although the long-range order does not persist forever, it may be well preserved during typical electron scattering time. Thus a temporary electron band structure emerges, and an associated elastic scattering does not contribute to the conduction (as in solid). This is in line with Edwards's 12] argument that one should deal with a local disorder "observed" by an electron along its mean free path, rather than with the global disorder. Therefore we suggest to subtract the elastic contribution from the total static structure factor $S_{\text {liq }}(q)$ in the liquid (e.g., 13, 14 ). Since in the liquid an electron couples directly to the ion number density, the elastic part must have the form [9]

$$
S_{\text {liq }}^{\prime}(\mathbf{q})=e^{-2 W}(2 \pi)^{3} n_{i} \sum_{\mathbf{G} \neq 0} \delta(\mathbf{q}-\mathbf{G}) .
$$

Then the "inelastic" part which determines the transport properties is $S_{\text {liq }}^{\prime \prime}(q)=S_{\text {liq }}(q)-S_{\text {liq }}^{\prime}(\mathbf{q})$. There may be various types of periodic structures in this regime, but they are very similar and we can use the bcc lattice. We have checked that the result is almost the same for face-centered cubic (fcc) and hexagonal close-packed (hcp) lattices.

At this stage we need to specify the matrix element of the elementary ei interaction $U_{\mathbf{q}, \sigma^{\prime} \sigma}$. Assuming the Coulomb potential screened by the static polarization of ideal, relativistic, strongly degenerate $\left(p \approx p^{\prime} \approx \hbar k_{F}\right)$ electrons, we obtain

$$
\frac{2 \pi N}{\hbar^{2}} \frac{1}{2} \sum_{\sigma \sigma^{\prime}}\left|U_{\mathbf{q}, \sigma^{\prime} \sigma}\right|^{2}=\frac{2 \pi N}{\hbar^{2} V^{2}} \frac{16 \pi^{2} Z^{2} e^{4}}{q^{4}|\varepsilon(q)|^{2}}\left(1-\frac{\hbar^{2} c^{2} q^{2}}{4 \epsilon_{F}^{2}}\right)
$$

where $\epsilon_{F}=\left[m_{e}^{2} c^{4}+p_{F}^{2} c^{2}\right]^{1 / 2}$ is the electron Fermi energy, $V$ is the normalization volume, and $\varepsilon(q)$ is the electron longitudinal static dielectric function [15].

The electric $(\sigma)$ and thermal $(\kappa)$ conductivities, and shear viscosity $(\eta)$ can be written in the form [2]

$$
\sigma=\frac{n_{e} e^{2}}{m_{e}^{*} \nu_{\sigma}}, \quad \kappa=\frac{\pi^{2} T n_{e}}{3 m_{e}^{*} \nu_{\kappa}}, \quad \eta=\frac{n_{e} m_{e}^{*} v_{F}^{2}}{5 \nu_{\eta}},
$$

where $m_{e}^{*}=\epsilon_{F} / c^{2}$, and

$$
\nu_{\sigma, \kappa, \eta}=4 \pi m_{e}^{*} Z^{2} e^{4} L_{\sigma, \kappa, \eta} n_{i} /\left(\hbar k_{F}\right)^{3}
$$




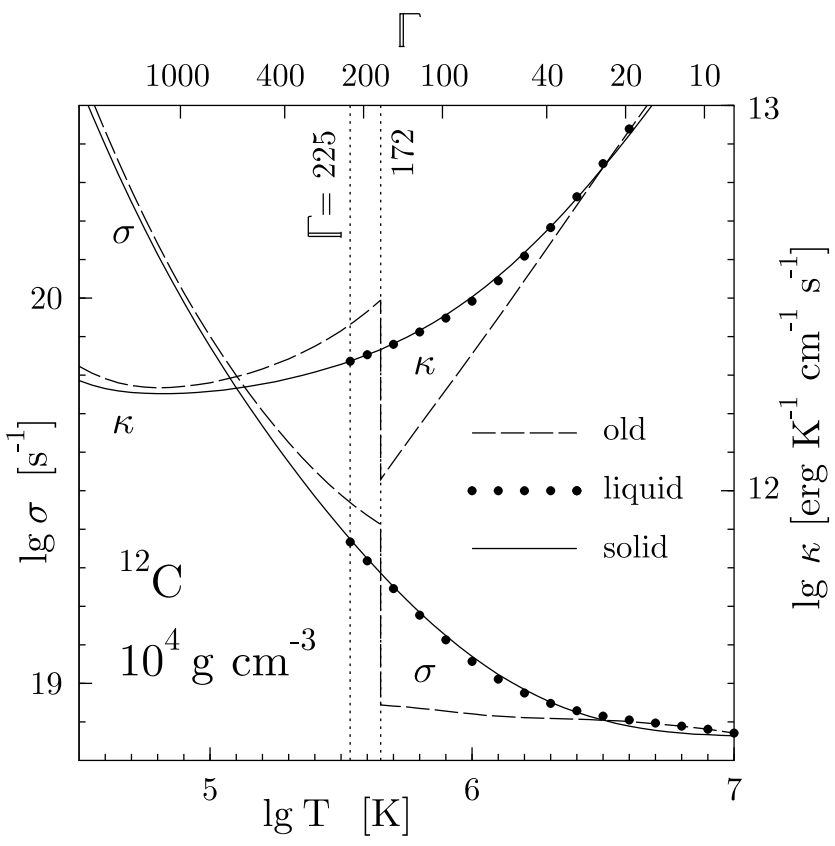

FIG. 1. Dependence of the electron electric (left vertical scale) and thermal (right vertical scale) conductivities of carbon plasma at density $10^{4} \mathrm{~g} \mathrm{~cm}^{-3}$ on temperature (lower horizontal scale) or ion coupling parameter (upper horizontal scale). Dashes are traditional results in ion liquid [3, ] and bcc crystal 4. 6. for $\Gamma_{m}=172$. Solid line is the present multi-phonon calculation in solid, extended artificially to high $T$; dots show the present calculation with reduced structure factor in liquid at $\Gamma \leq 225$. Vertical dotted lines correspond to $\Gamma=172$ and 225 .

are the effective collision frequencies. Here, the effective Coulomb logarithms are

$$
\begin{aligned}
L_{\sigma, \kappa}= & \int_{q_{0}}^{2 k_{F}} \frac{\mathrm{d} q q^{3}}{q^{4}|\varepsilon(q)|^{2}}\left[1-\frac{1}{4}\left(\frac{\hbar q}{m_{e}^{*} c}\right)^{2}\right] S_{\sigma, \kappa}(q), \\
L_{\eta}= & 3 \int_{q_{0}}^{2 k_{F}} \frac{\mathrm{d} q q^{3}}{q^{4}|\varepsilon(q)|^{2}}\left(1-\frac{q^{2}}{4 k_{F}^{2}}\right) \\
& \times\left[1-\frac{1}{4}\left(\frac{\hbar q}{m_{e}^{*} c}\right)^{2}\right] S_{\sigma}(q),
\end{aligned}
$$

$q_{0}=0$ for the liquid phase and $q_{0}=q_{B}$ for the solid phase [6], and $S_{\sigma, \kappa}(q)$ are the effective static structure factors. In the liquid regime, we approximate $S_{\sigma, \kappa}(q)$ by $S_{\text {liq }}^{\prime \prime}(q)$ as described above. In the solid regime, we have

$$
\begin{aligned}
S_{\sigma}(q) & =\frac{1}{2 \pi} \int_{-\infty}^{+\infty} \mathrm{d} \omega \int_{-\infty}^{+\infty} \mathrm{d} t \frac{e^{-i \omega t} z}{1-e^{-z}} S_{\mathrm{sol}}^{\prime \prime}(\mathbf{q}, t), \\
S_{\kappa}(q) & =S_{\sigma}(q)+\left(\frac{3 k_{F}^{2}}{q^{2}}-\frac{1}{2}\right) \delta S_{\kappa}(q), \\
\delta S_{\kappa}(q) & =\frac{1}{2 \pi} \int_{-\infty}^{+\infty} \mathrm{d} \omega \int_{-\infty}^{+\infty} \mathrm{d} t \frac{e^{-i \omega t} z^{3}}{1-e^{-z}} S_{\mathrm{sol}}^{\prime \prime}(\mathbf{q}, t),
\end{aligned}
$$

with $z=\hbar \omega / T$. The integration over $\omega$ can be performed analytically. The remaining numerical integration over $t$ is then facilitated by shifting the integration path in complex plane: $t=t^{\prime}-i \hbar / 2 T$, where $t^{\prime}$ is real. The final result reads:

$$
\begin{aligned}
S_{\sigma}(q) & =\frac{1}{2} \int_{-\infty}^{+\infty} \frac{\mathrm{d} x}{\cosh ^{2} x} e^{-2 W} K\left(q, T, t^{\prime}\right), \\
\delta S_{\kappa}(q) & =\int_{-\infty}^{+\infty} \mathrm{d} x \frac{1-2 \sinh ^{2} x}{\cosh ^{4} x} e^{-2 W} K\left(q, T, t^{\prime}\right),
\end{aligned}
$$

where $x=\pi t^{\prime} T / \hbar$ and

$$
K\left(q, T, t^{\prime}\right)=\exp \left[\frac{\hbar q^{2}}{2 M}\left\langle\frac{\cos \left(\omega_{\nu} t^{\prime}\right)}{\omega_{\nu} \sinh \left(z_{\nu} / 2\right)}\right\rangle_{\mathrm{ph}}\right]-1 .
$$

Retaining the term $\propto q^{2}$ in the expansion of $K(q, T, t)$, we recover the standard one-phonon approximation $[36$.

Figures 11 and 2 show temperature dependence of the electric and thermal conductivities for carbon plasma at density $10^{4} \mathrm{~g} \mathrm{~cm}^{-3}$ and for iron plasma at $10^{8} \mathrm{~g} \mathrm{~cm}^{-3}$, respectively, calculated in the Born approximation. In spite of large differences in densities and chemical elements, the figures are fairly similar. Dashes show the traditional results calculated with the full structure factor $S_{\text {liq }}(q)$ in ion liquid [3.7] and in the one-phonon approximation [4] 6] for bcc crystal (notice that the results of ref. [6] for fcc crystals are in error; actually, they are very similar to those for bcc). One can see strong jumps of $\kappa$ and $\sigma$ at the melting point $\Gamma_{m}=172$. Solid lines are the present results in the solid phase (including multi-phonon processes), while dots show the present results in the liquid obtained using the analytic fits for the static structure factor $S_{\text {liq }}(q)$ at $\Gamma \leq 225$ [14] by subtracting the longrange correlations (see above). For illustration (as suggested by H.E. DeWitt), we have extended the improved results in liquid by shifting artificially the melting point to lower $T$ (to $\Gamma=225$, considering thus supercooled liquid) and the improved results in solid by shifting the melting to higher $T$ (lower $\Gamma$, superheated crystal). The curves for liquid and solid ions match one another quite well, and the jumps at the melting point actually disappear. In a wide temperature range the improved curves for liquid and solid almost coincide. We have verified that the same is true for all transport coefficients (including shear viscosity) in wide range of densities for a number of chemical elements. Thus one can observe that electron transport properties in SCCP of ions appear to be fairly insensitive to the state of SCCP (liquid or solid, bcc or other crystals). This should be taken into account in various astrophysical applications, for instance, in calculating the temperature growth from the surface into interior of the isolated neutron stars important for theories of neutron star cooling [16, ], The thermal conductivity of neutron-star envelopes to be reconsidered lies exactly in the "sensitivity strip" [16] which strongly affects the temperature profiles and neutron-star cooling. 


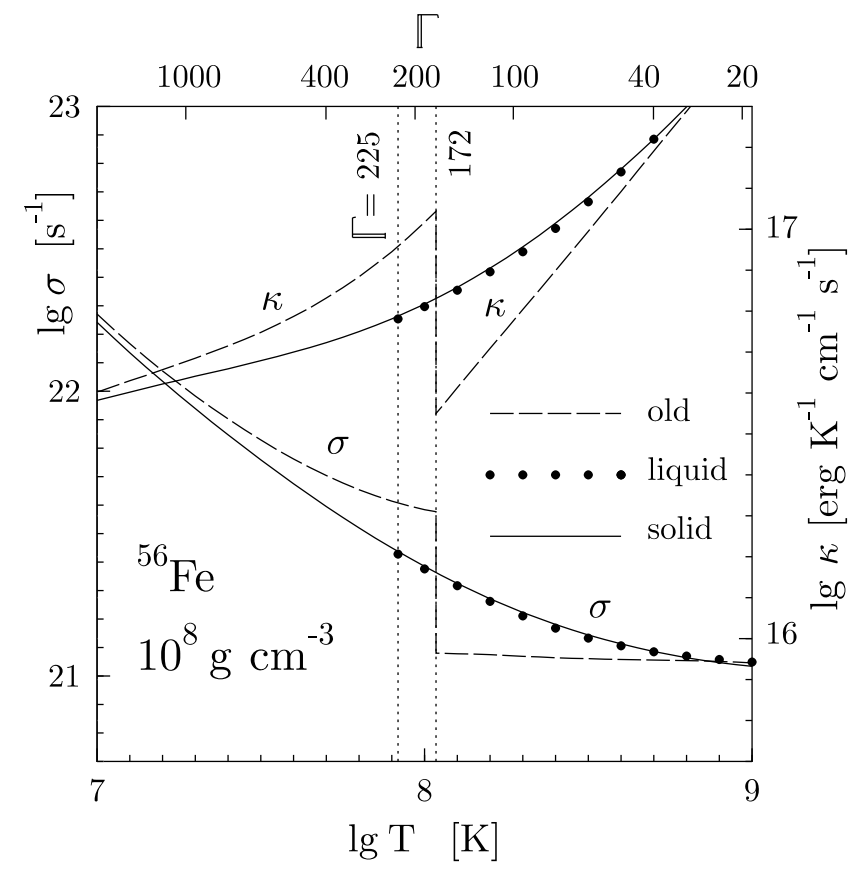

FIG. 2. Same as in Fig. 11 but for iron matter at density $10^{8} \mathrm{~g} \mathrm{~cm}^{-3}$.

We are grateful to H.E. DeWitt and F.J. Rogers for useful discussions. This work was supported in part by RFBR (grant 96-02-16870a), RFBR-DFG (grant 96-0200177G), and INTAS (grant 96-0542).

[1] H. Nagara, Y. Nagata, and T. Nakamura, Phys. Rev. A 36, 1859 (1987)

[2] E. Flowers and N. Itoh, Astrophys. J. 206, 218 (1976)

[3] N. Itoh, Y. Kohyama, N. Matsumoto, and M. Seki, Astrophys. J. 285, 758 (1984)

[4] N. Itoh, H. Hayashi, and Y. Kohyama, Astrophys. J. 418, 405 (1993); 436, 418 (E) (1994)

[5] N. Itoh, in The Equation of State in Astrophysics, edited by G. Chabrier and E. Schatzman (Cambridge University Press, Cambridge, 1994), p. 394

[6] D.A. Baiko and D.G. Yakovlev, Astron. Lett. 21, 702 (1995)

[7] A.Y. Potekhin, G. Chabrier, and D.G. Yakovlev, Astron. Astrophys. 323, 415 (1997)

[8] E.L. Pollock and J.P. Hansen, Phys. Rev. A 8, 3110 (1973)

[9] C. Kittel, Quantum Theory of Solids (Wiley, New York, 1963)
[10] M.E. Raikh and D.G. Yakovlev, Astrophys. Sp. Sci. 87, 193 (1982)

[11] P. Schmidt, G. Zwicknagel, P.G. Reinhard, C. Toepffer, Phys. Rev. E 56, 7310 (1997)

[12] S.F. Edwards, Proc. R. Soc. A 267, 518 (1962)

[13] J.P. Hansen, Phys. Rev. A 8, 3096 (1973)

[14] D.A. Young, E.M. Corey, and H.E. DeWitt, Phys. Rev. A 44, 6508 (1991)

[15] B. Jancovici, Nuovo Cim. 25, 428 (1962)

[16] E.H. Gudmundsson, C.J. Pethick, and R.I. Epstein, Astrophys. J. 272, 286 (1983) 